EPiC Series in Engineering
Volume 3, 2018, Pages 1184-1192
HIC 2018. 13th International
Conference on Hydroinformatics

\title{
Drainage network modelling with a novel algorithm for junction calculation
}

\author{
Qian Li' ${ }^{1}$ Xilin Xia², Qiuhua Liang², Wen Xiao' \\ ${ }^{1}$ School of Engineering, Newcastle University, Newcastle upon Tyne, NE1 7RU, \\ UK \\ ${ }^{2}$ School of Architecture, Building and Civil Engineering, Loughborough University, \\ LE11 3RU, UK \\ Corresponding author:Q.Li26@newcastle.ac.uk
}

\begin{abstract}
Most of the existing drainage network models focus on capturing different flow regimes in sewer pipes. Despite being important to produce reliable results of urban flood simulation, the development of robust scheme to describe the coupled conditions at pipe-to-pipe intersections has received less research attention. In this work, the two-component pressure approach (TPA) is chosen to support pipe flow calculation due to its superior capability in simulating complicated transient flow between the free-surface and pressurized conditions in pipes. To avoid the complicated boundary conditions as required by a TPA model to approximate the junction connections, a novel strategy is proposed in this work where the flow at a junction is calculated using the 2D shallow water equations (SWEs). In junctions, irregular grids are created automatically according to the layout of connecting pipes, on which a first-order Godunov-type finite volume scheme is implemented to solve the 2D SWEs to simulate the junction flow. The 2D SWEs model is driven by the boundary conditions provided from the pipe calculations and rainfall input in necessary, which in turn creates boundary conditions for pipe calculations at the next time step. It is expected that the new approach as proposed will support largescale drainage network modelling with higher efficiency and stability.
\end{abstract}

Keywords: Drainage network; Junction calculation; TPA; Shallow water equations; Transient flow.

\section{Introduction}

With increasing frequency of urban flooding during summers or wet seasons around the world, more researches have been conducted to develop tools to understand and predict the dynamic urban flood inundation process. Particularly, urban overland flow modelling [1] and dual drainage modelling [2] have received considerable attention and substantial research progress has been made in recent years. However, accurate simulation of urban drainage systems is still a challenging task due to the complex transient flow dynamics in both pipes and junctions. 
Different numerical methods have been developed and used to predict flow dynamics in drainage networks. The approach adopted by the storm water management model (SWMM) to calculate the linknode flows is to solve the continuity equation at junctions to update water depth and approximate the dynamic fluid equations in each pipe using a simple finite difference scheme. However, the adopted numerical method is ineffective in capturing the highly unsteady flows and the transient flows between the free-surface and pressurized conditions as frequently encountered during urban floods induced by intense rainfall events. The Pressman slot scheme proposed by Cunge [3] has been widely used in transient free-surface and pressurized flow simulations. But according to Vasconcelos [4], choosing an appropriate slot width is difficult when implementing the Pressman slot scheme and the use of inappropriate slot width may lead to model instability. Subsequently Vasconcelos and Wright [4-5] proposed a two-component pressure approach (TPA) to overcome this numerical limitation, which has been applied to simulate storm sewer networks [6]. However, when calculating junction flows, the TPA model requires complicated boundary conditions, which are difficult to implement and directly affect the model's computational efficiency and stability.

In this paper, a model based on TPA is developed for drainage network modelling with an innovative strategy proposed to improve junction calculations. The proposed approach treats a junction as a $2 \mathrm{D}$ domain with the flow approximated by an irregular mesh based model solving the 2D shallow water equations (SWEs), removing the necessity of any complicated boundary settings.

\section{Drainage network model}

\subsection{Pipe model}

\subsubsection{TPA governing equations}

For a prismatic channel such as a pipe without lateral inflow or outflow, the governing equations of a TPA model that simulates both free-surface and pressurized flows may be written in the form of 1D conservation laws as:

$$
\begin{gathered}
\frac{\partial \mathbf{U}_{P}}{\partial t}+\frac{\partial \mathbf{F}_{P}}{\partial x}=\mathbf{S}_{P b}+\mathbf{S}_{P f} \\
\mathbf{U}_{P}=\left[\begin{array}{c}
A \\
Q_{P}
\end{array}\right], \mathbf{F}_{P}=\left[\begin{array}{c}
Q_{P} \\
Q_{P}^{2} / A+I
\end{array}\right], \mathbf{S}_{P b}=\left[\begin{array}{c}
0 \\
-g A \frac{d z}{d x}
\end{array}\right], \mathbf{S}_{P f}=\left[\begin{array}{c}
0 \\
-c_{D} \frac{P Q_{P}\left|Q_{P}\right|}{A^{2}}
\end{array}\right]
\end{gathered}
$$

where subscripts $\mathrm{P}, \mathrm{b}$ and f respectively represent 'pipe', 'bed slop' and 'friction slop'; $\mathrm{t}$ denotes time; $x$ is the distance along the pipe under consideration; $A$ is the cross-sectional area of the water flow; $Q_{P}$ is the flow discharge; $\mathrm{z}$ is the bottom elevation of the pipe above an arbitrary datum; $c_{D}=g n_{P}^{2} R_{P}^{-1 / 3}$ is the roughness coefficient with $n_{p}$ being the pipe Manning coefficient and $R_{P}$ being the hydraulic radius; $P$ is the wetted perimeter; $I$ is the pressure term, which can be expressed as $I=p A / \rho$ under the freesurface flow conditions with $p$ being the fluid pressure at the centroid of cross-sectional area and $\rho$ being the fluid density. While under pressurized condition, a different pressure force related to surcharge head can be obtained based on the assumption of elastic property of pipe wall. Therefore, I may be calculated by

$$
I\left(h_{P}, H\right)=\left\{\begin{array}{cc}
\frac{1}{24}\left[3 \sin (\theta / 2)-\sin ^{3}(\theta / 2)-3(\theta / 2) \cos (\theta / 2)\right] g d^{3} & \text { if ventilated } \\
\frac{\pi}{4} g d^{2}(H+d / 2) & \text { if pressurized }
\end{array}\right.
$$


where $h_{P}$ is the water depth in the pipe; $d$ is the pipe diameter; $\theta$ is the wetted angle in the pipe; $g$ is the acceleration due to gravity; $H$ is the pressurized head calculated by

$$
H=\frac{a^{2}}{g}\left(\frac{A-A_{P}}{A_{P}}\right)
$$

in which $a$ is the acoustic wave speed and $A_{p}$ is the original cross-sectional area of the pipe.

For a circular pipe, under the free surface conditions the variables $h, A, P$ and the top width $T$ are functions of the wetted angle $\theta$ given by [6]:

$$
\begin{gathered}
h_{P}=\frac{1}{2}\left[1-\cos \left(\frac{\theta}{2}\right)\right] d \\
A=\frac{1}{8}(\theta-\sin \theta) d^{2} \\
P=\frac{1}{2} \theta d \\
T=d \sin (\theta / 2)
\end{gathered}
$$

The speed of gravity waves in a pipe is defined as:

$$
c=\left\{\begin{array}{cc}
\sqrt{\frac{g A}{T}}=\sqrt{\frac{g d(\theta-\sin \theta)}{8 \sin (\theta / 2)}} & \text { if ventilated } \\
\mathrm{a} & \text { if pressurized }
\end{array}\right.
$$

The above 1D TPA governing equations are numerally solved using a first-order Godunov-type finite volume scheme. The 1D computational domain (each of the pipes) is discretised using uniform grids. In an arbitrary cell, the flow variables are advanced from time level $n$ to $n+1$ using the following explicit finite volume time-marching formula:

$$
\mathbf{U}_{P i}^{n+1}=\mathbf{U}_{P i}^{n}-\frac{\Delta t}{\Delta x}\left[\mathbf{F}_{i+1 / 2}^{n}-\mathbf{F}_{i-1 / 2}^{n}\right]+\Delta t\left(\mathbf{S}_{P b i}^{n}+\mathbf{S}_{P f i}^{n+1}\right)
$$

where subscript $i$ is the cell index; $\Delta x$ is the cell length; $\mathbf{F}_{i+1 / 2}^{n}$ and $\mathbf{F}_{i-1 / 2}^{n}$ are the numerical fluxes across the right and left cell interface evaluated using HLL Riemann solver scheme [7]; the bed slop terms in $\mathbf{S}_{P b i}^{n}$ are simply estimated using a central difference scheme; and the friction source terms in $\mathbf{S}_{P f i}^{n+1}$ are discretised using an implicit scheme detailed in the following section.

\subsubsection{TPA friction discretization scheme}

The momentum equation of (10) can be rewritten as

$$
Q_{P i}^{n+1}=Q_{P i}^{n}+\Delta t\left(F_{P i}^{n}+S_{P f i}^{n+1}\right)
$$

with $F_{P i}^{n}=-\frac{1}{\Delta x}\left[F_{i+1 / 2}^{n}-F_{i-1 / 2}^{n}\right]+S_{P b i}^{n}$, and $S_{P f i}^{n+1}=-g n_{P}^{2}\left(P_{i}^{n}\right)^{\frac{4}{3}} Q_{P i}^{n+1}\left|Q_{P i}^{n+1}\right| /\left(A_{i}^{n}\right)^{\frac{7}{3}}$

Since $A_{i}^{n}$ is a function of water depth, the friction term may become excessively large when the depth becomes small, which may subsequently cause numerical instability. To ensure stable simulations under various flow conditions including wetting and drying, the fully implicit discretization scheme proposed by $\mathrm{Xia}[1]$ is adopted in this work. The scheme involves defining several auxiliary variables as follows:

$$
\begin{aligned}
U_{P i}^{n+1} & =Q_{P i}^{n+1} / A_{i}^{n} \\
U_{P i}^{n} & =Q_{P i}^{n} / A_{i}^{n} \\
\bar{F}_{P i}^{n} & =F_{P i}^{n} / A_{i}^{n}
\end{aligned}
$$




$$
\bar{S}_{P f i}^{n+1}=\frac{S_{P f}^{n+1}}{A_{i}^{n}}=\frac{-g n_{P}^{2}\left(P_{i}^{n}\right)^{\frac{4}{3}} U_{P i}^{n+1}\left|U_{P i}^{n+1}\right|}{\left(A_{i}^{n}\right)^{\frac{4}{3}}}
$$

with these auxiliary variables and further defining $\alpha_{L}=-g n_{P}^{2}\left(P_{i}^{n}\right)^{\frac{4}{3}} /\left(A_{i}^{n}\right)^{\frac{4}{3}}$, the discretized momentum equation (11) can be rewritten as

$$
\alpha_{L} \Delta t U_{P i}^{n+1}\left|U_{P i}^{n+1}\right|-U_{P i}^{n+1}+U_{P i}^{n}+\Delta t \bar{F}_{P i}^{n}=0
$$

The equation is then solved using Newton-Raphson iteration method and $Q_{P i}^{n+1}$ can be retrieved from $U_{P i}^{n+1}$ according to the relationship as defined above. The iteration procedure is implemented as follow:

$$
U_{P}^{k+1}=U_{P}^{k}+\frac{\alpha_{L} \Delta t U_{P}^{k}\left|U_{P}^{k}\right|+\Delta t \bar{F}_{P i}^{n}+U_{P i}^{n}-U_{P}^{k}}{\left(1-2 \alpha_{L} \Delta t\left|U_{P}^{k}\right|\right)}
$$

where $k$ denotes the iteration step and the following convergence criterion is adopted:

$$
\left|U_{P}^{k+1}-U_{P}^{k}\right| \leq 0.001\left|U_{P}^{k}\right|
$$

\subsection{Junction model}

A junction is a node in a drainage system connecting one or more pipes and free surface conditions commonly apply to the junction flow. In this work, a new modelling strategy is proposed to idealize the junction as 2D domains, subsequently the junction flow can be depicted using a fully 2D SWEs model to avoid the complicated boundary conditions as required by TPA model [6].

\subsubsection{D SWEs}

Fig. 1 illustrates a schematic diagram for a junction connecting three pipes. The diameter of each pipe is denoted by $d_{i}(i=1,2,3)$; $\mathrm{P} 1$ and $\mathrm{P} 2$ are assumed to be inflow pipes while $\mathrm{P} 3$ is the outflow pipe. Based on the layout of the inflow and outflow pipes, the junction domain is approximated using an irregular grid cell as indicated in the second graph. Noted that the "shape" of the junction cell is changed but it does not affect the calculation, which will be detailed in the section 2.2.2. With this gridding approach, a cell-centred finite volume numerical scheme may be implemented to solve the SWEs and capture the flow dynamics in the junction. In this case, the inflow from the two incoming pipes (P1 and P2) is mixed in the junction and then discharge into the outflow pipe (P3). As shown in the last graph, the cell edges connected with pipes are defined as 'open' faces, through which the inflow $\left(q_{1}\right.$ and $q_{2}$ )/outflow $\left(q_{3}\right)$ from the connecting pipes are imposed as boundary conditions for the $2 \mathrm{D}$ junction flow calculation. In order to reinforce strict conservation of mass and momentum at the connected interface between pipes and junction, the fluxes obtained from the TPA calculation are directly used to provide interface fluxes onto the $2 \mathrm{D}$ domain. The $2 \mathrm{D}$ cell edges without connecting to a pipe are set to be 'closed', with no-flow boundary conditions imposed. 


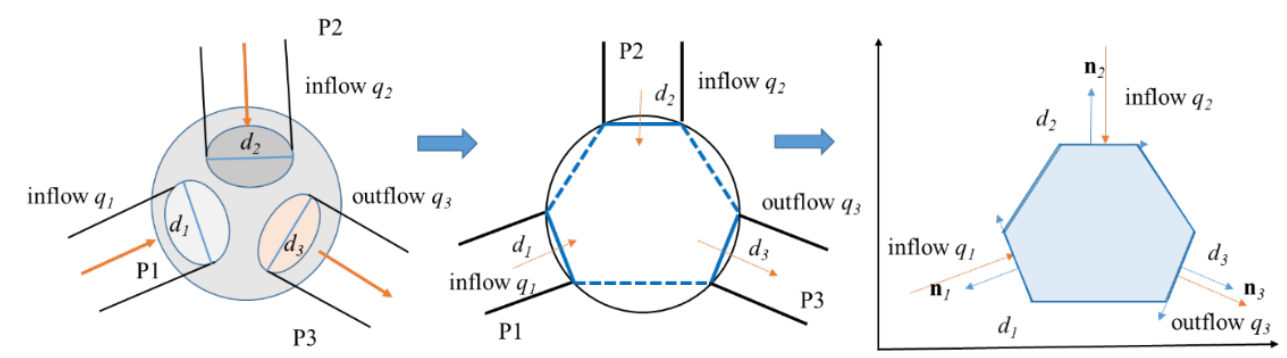

Figure 1 Spatial discretization scheme for a junction

The 2D SWEs describing the free-surface flow in a junction may be written in a matrix form as

$$
\begin{gathered}
\frac{\partial \mathbf{U}_{J}}{\partial t}+\frac{\partial \mathbf{F}_{J}}{\partial x}+\frac{\partial \mathbf{G}_{J}}{\partial y}=\mathbf{R}+\mathbf{S}_{J b}+\mathbf{S}_{J f} \\
\mathbf{U}_{J}=\left[\begin{array}{c}
h_{J} \\
u_{x} h_{J} \\
u_{y} h_{J}
\end{array}\right], \mathbf{F}_{J}=\left[\begin{array}{c}
u_{x} h_{J} \\
u_{x}^{2} h_{J}+\frac{1}{2} g h_{J}^{2} \\
u_{x} u_{y} h_{J}
\end{array}\right], \mathbf{G}_{J}=\left[\begin{array}{c}
u_{y} h_{J} \\
u_{x} u_{y} h_{J} \\
u_{y}^{2} h_{J}+\frac{1}{2} g h_{J}^{2}
\end{array}\right], \\
\mathbf{R}=\left[\begin{array}{c}
R \\
0 \\
0
\end{array}\right], \mathbf{S}_{J b}=\left[\begin{array}{c}
0 \\
0 \\
-g h_{J} \frac{\partial z_{b}}{\partial x} \\
-g h_{J} \frac{\partial z_{b}}{\partial y}
\end{array}\right], \text { and } \mathbf{S}_{J f}=\left[\begin{array}{c} 
\\
-\frac{\tau_{b x}}{\rho} \\
-\frac{\tau_{b y}}{\rho}
\end{array}\right]
\end{gathered}
$$

where subscript $J$ represents junction; $h_{J}$ is the water depth in junction area; $u_{x}$ and $u_{y}$ are the two depth-averaged velocities along the $x$ - and $y$-directions, respectively; $q_{x}\left(=u_{x} h_{J}\right)$ and $q_{y}\left(=u_{y} h_{J}\right)$ are the corresponding unit-width discharges; $\tau_{b x}$ and $\tau_{b y}$ are bed friction stresses calculated by $\tau_{b x}=\rho C_{f} u_{x} \sqrt{u_{x}^{2}+u_{y}^{2}}$ and $\tau_{b y}=\rho C_{f} u_{y} \sqrt{u_{x}^{2}+u_{y}^{2}}$, where $C_{f}=g n_{J}^{2} / h_{J}^{1 / 3}$ is the bed roughness coefficient with $n_{J}$ being the junction Manning coefficient.

\subsubsection{Numerical scheme for junctions}

To construct the proposed 2D junction model, the above governing equations are solved using a firstorder Godunov-type finite volume scheme. The resulting 2D time-marching formula is written as

$$
\mathbf{U}_{J}^{n+1}=\mathbf{U}_{J}^{n}-\frac{\Delta t}{\Omega_{i}} \sum_{K=1}^{N} \mathbf{P}_{K}\left(\mathbf{U}_{J}^{n}\right) l_{K}+\Delta t\left(\mathbf{R}_{i}^{n}+\mathbf{S}_{J b i}^{n}+\mathbf{S}_{J f i}^{n+1}\right)
$$

where $\Omega_{i}$ is the cell area (hence it is independent of the cell shape), $K$ is the edge index and $l_{K}$ is the length of that edge, $\quad \mathbf{P}_{K}\left(\mathbf{U}_{J}^{n}\right)=\mathbf{F}_{J K}\left(\mathbf{U}_{J}^{n}\right) n_{x}+\mathbf{G}_{J K}\left(\mathbf{U}_{J}^{n}\right) n_{y}$ with $\mathbf{n}=\left(n_{x}, n_{y}\right)$ being the unit outward vector normal to edge $K$. It should be noted that the fluxes through the connecting edges between pipes and junction have already been calculated by the pipe TPA model and will be directly used here to complete the junction calculation. 


\subsubsection{D friction term discretization scheme}

The momentum components of the 2D SWEs are given as follows:

$$
\mathbf{Q}_{J i}^{n+1}=\mathbf{Q}_{J i}^{n}+\Delta t\left(\mathbf{B}_{i}^{n}+\mathbf{S}_{J f}^{n+1}\right)
$$

where $\mathbf{B}_{i}^{n}=-\frac{1}{\Omega_{i}} \sum_{K=1}^{N} \mathbf{P}_{K}\left(\mathbf{U}_{J}^{n}\right) l_{K}+\mathbf{S}_{J b i}^{n}$. Specifically, the friction source terms are given by:

$$
\mathbf{S}_{J f i}^{n+1}=\frac{-g n_{J}^{2} \mathbf{Q}_{J i}^{n+1}\left|\mathbf{Q}_{J i}^{n+1}\right|}{\left(h_{J i}^{n}\right)^{\frac{7}{3}}}
$$

Similar to the 1D scheme, auxiliary variables are introduced and defined as follows:

$$
\begin{aligned}
\mathbf{U}_{J i}^{n+1} & =\frac{\mathbf{Q}_{J i}^{n+1}}{h_{J i}^{n}} \\
\mathbf{U}_{J i}^{n} & =\frac{\mathbf{Q}_{J i}^{n}}{h_{J i}^{n}} \\
\overline{\mathbf{B}}_{i}^{n} & =\frac{\mathbf{B}_{i}^{n}}{h_{J i}^{n}}
\end{aligned}
$$

Then the friction source terms can be rewritten as:

$$
\overline{\mathbf{S}}_{J f i}^{n+1}=\frac{-g n_{J}^{2} \mathbf{U}_{J i}^{n+1}\left|\mathbf{U}_{J i}^{n+1}\right|}{\left(h_{J i}^{n}\right)^{\frac{4}{3}}}
$$

This leads to a new time-marching formula for $\mathbf{U}$ given as follow:

$$
\mathbf{U}_{J i}^{n+1}=\mathbf{U}_{J i}^{n}+\Delta t\left(\overline{\mathbf{B}}_{i}^{n}+\overline{\mathbf{S}}_{J f i}^{n+1}\right)
$$

The Newton-Raphson method is used to solve this implicit equation and the solution procedure is given by:

$$
\mathbf{U}_{J}^{k+1}=\mathbf{U}_{J}^{k}+\left[\mathbf{I}-\Delta t \mathbf{J}\left(\mathbf{U}_{J}^{k}\right)\right]^{-1}\left[\Delta t \overline{\mathbf{S}}_{J f}\left(\mathbf{U}_{J}^{k}\right)+\Delta t \overline{\mathbf{B}}_{i}^{n}-\left(\mathbf{U}_{J}^{k}-\mathbf{U}_{J}^{n}\right)\right]
$$

where $\mathbf{I}$ is the identity matrix, $k$ represents the iteration step, $\mathbf{U}_{J}^{n}$ contains the initial values and $\mathbf{J}$ is the Jacobian matrix of $\overline{\mathbf{S}}_{J f}$ defined as:

$$
\mathbf{J}\left(\mathbf{U}_{J}^{k}\right)=\left[\begin{array}{ll}
\frac{\partial \bar{S}_{J f x}}{\partial U_{J x}^{k}} & \frac{\partial \bar{S}_{J f x}}{\partial U_{J y}^{k}} \\
\frac{\partial \bar{S}_{J j y}}{\partial U_{J x}^{k}} & \frac{\partial \bar{S}_{J f y}}{\partial U_{J y}^{k}}
\end{array}\right]=\left[\begin{array}{ll}
\frac{\alpha_{J}\left(U_{J x}^{k}\right)^{2}}{\sqrt{\left(U_{J x}^{k}\right)^{2}+\left(U_{J y}^{k}\right)^{2}}} & \frac{\alpha_{J} U_{J x}^{k} U_{J y}^{k}}{\sqrt{\left(U_{J x}^{k}\right)^{2}+\left(U_{J y}^{k}\right)^{2}}} \\
\frac{\alpha_{J} U_{J x}^{k} U_{J y}^{k}}{\sqrt{\left(U_{J x}^{k}\right)^{2}+\left(U_{J y}^{k}\right)^{2}}} & \frac{\alpha_{J}\left(U_{J y}^{k}\right)^{2}}{\sqrt{\left(U_{J x}^{k}\right)^{2}+\left(U_{J y}^{k}\right)^{2}}}
\end{array}\right]
$$

where $\alpha_{J}=-g n_{J}^{2} /\left(h_{J i}^{n}\right)^{\frac{4}{3}}$

and the convergence criterion is defined in this work as:

$$
\left|\mathbf{U}_{J}^{k+1}-\mathbf{U}_{J}^{k}\right| \leq 0.001\left|\mathbf{U}_{J}^{k}\right|
$$

\section{Results and discussion}

In order to validate the current pipe-junction model and demonstrate its performance for pipe network simulation, a simple test is considered and simulated, which involves one pipe linking to two junctions, 
as illustrated in Fig. 2. The circular pipe is $30 \mathrm{~m}$ in length and $1 \mathrm{~m}$ in diameter. The junctions are $2 \mathrm{~m}$ in diameter. The whole pipe network system is assumed to be horizontal and frictionless. The simulation lasts $100 \mathrm{~s}$. The initial water depth is $0.5 \mathrm{~m}$ in the pipe and $0.6 \mathrm{~m}$ and $0.4 \mathrm{~m}$ respectively in the two junctions. Closed boundary conditions are imposed in the two junctions.

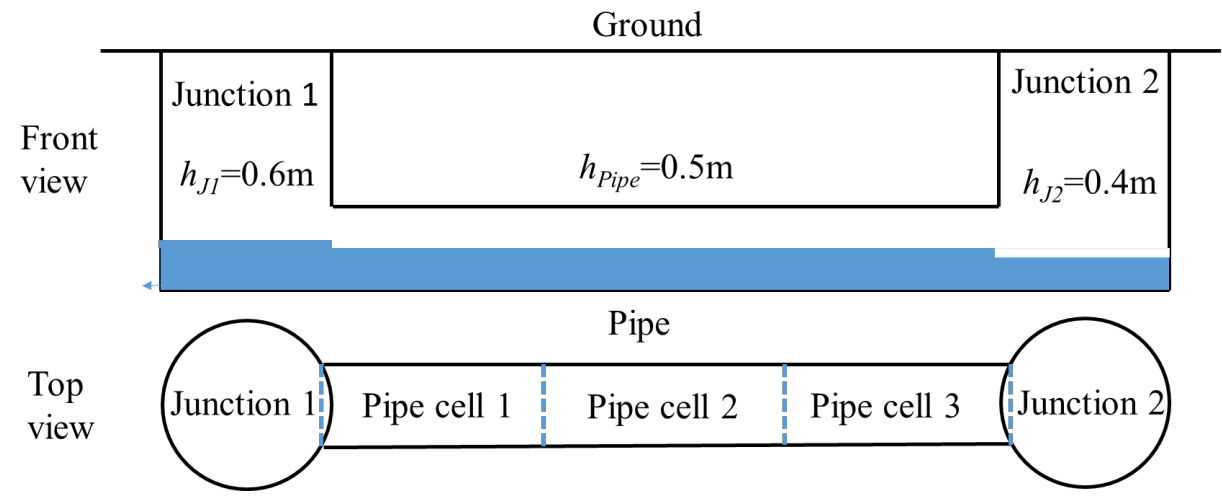

Figure 2 An idealised pipe network with one pipe and two junctions
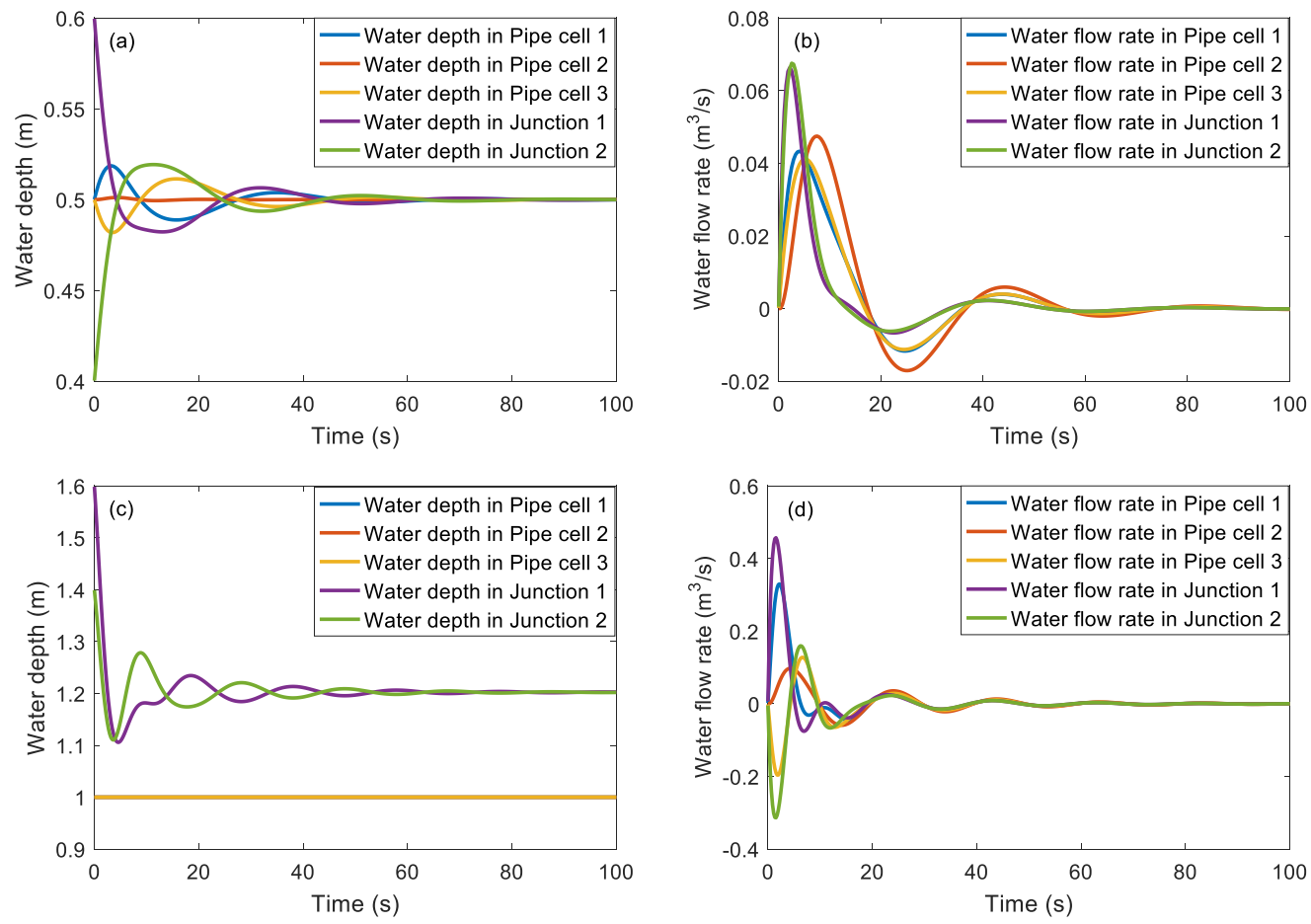

Figure 3 Simulation of water depth and water flow rate under free-surface condition ((a) and (b)) and pressurized condition ((c) and (d)) 
Fig. 3(a) shows the variation of water depth in each of the two junctions and each of the three pipe cells during the simulation. As the simulation starts, the water depth in Junction 1 is $0.1 \mathrm{~m}$ and $0.2 \mathrm{~m}$ higher respectively than the depth in the pipe and Junction 2 . The unequal water surface subsequently creates disturbance to the system which propagates back and forth between two junctions and finally settles down to a steady water depth of $0.5 \mathrm{~m}$, which is as expected. Fig. 3(a) presents symmetrical graph with water depth varying around $0.5 \mathrm{~m}$, which is consistent with the initial conditions. Fig. 3(b) presents the change of flow rates in the pipe and junctions. The flow rates in Junction 1 and Junction 2 are similar due to the same initial water elevation difference. The flow rates in junctions increase sharply at the beginning and reach a peak at $0.06756 \mathrm{~m}^{3} / \mathrm{s}$ at $\mathrm{t}=2.559 \mathrm{~s}$, and then descend quickly to become zero. The flow rates in pipe cells also vary dramatically at the beginning and then settle down to zero quickly. The difference existing in the flow rate variation curves between different pipe cells reflects the wave propagation process inside the pipe. Variation of mass and momentum has also been recorded and monitored during the simulation and mass and momentum conservation have been verified and confirmed for the current pipe-junction model.

To further test the model for pressurized flow simulation, the initial water depth in Junction 1, pipe and Junction 2 is modified to be $1.6 \mathrm{~m}, 1 \mathrm{~m}$ and $1.4 \mathrm{~m}$, respectively. So the pipe is full at the beginning. Fig. 3 (c) shows the variation of water depth in pipe and junctions. The pipe remains to be full and hence the water depth is equal to pipe diameter. Head difference in the two junctions creates a flow into the pipe, which then oscillates back and forth until an equilibrium condition is reached in this system. It should be noted that a TPA model allows a pipe to slightly expand under the pressurized condition, which allows extra mass to fill the expanded volume. This subsequently leads to slightly lower steady-state water depth in the junctions, compared with the theoretical value of $1.5 \mathrm{~m}$. As shown in Fig. 3(d), the flows in Junction 1 and 2 have the opposite directions and the peak flow rate in Junction 1 is higher than that in Junction 2 due to a higher head. The flow rates in pipe cells vary accordingly with the change of the rates in the junctions. The flow rates gradually become zero in all of the cells and the water surface also becomes steady.

\section{Conclusions}

This paper introduces a new numerical method to approximate the junction nodes in drainage network modelling. Assuming free-surface flow under the atmospheric pressure in junctions, the flow conditions inside a junction can be then simulated using a 2D SWEs model, which provides an effective way to predict the junction flows and avoid the complicated boundary conditions as required by a TPA model. To solve the 2D governing equations at a junction, irregular meshes are created according to pipejunction connection configurations, on which a first-order Godunov-type finite volume scheme is implemented to update the flow variables. The boundary conditions to the 2D domain are provided from the pipe calculations.

For the flows in pipes, a TPA model is adopted to solve the 1D governing equations using a first-order Godunov-type finite volume scheme. The 1D TPA model is dynamically coupled to the 2D junction model via boundary conditions, effectively providing a new robust pipe network model.

The performance of the new pipe network modelling strategy is successfully demonstrated through the simulation of a pipe network with a simplified configuration. The proposed new pipe network model is expected to provide enhanced computational efficiency and stability for large-scale drainage network modelling. 


\section{References}

[1] Xia, X., Liang, Q., Ming, X., Hou, J, An efficient and stable hydrodynamic model with novel source term discretization schemes for overland flow and flood simulations, Water Resources Research, 2017.

[2] Li, W., Chen, Q., \& Mao, J, Development of 1D and 2D coupled model to simulate urban inundation: an application to Beijing Olympic Village. Chinese Science Bulletin, 2009, 54(9), 1613-1621.

[3] Cunge, J. A., Holly, F. M., \& Verwey, A Practical aspects of computational river hydraulics, 1980.

[4] Vasconcelos, J. G., Wright, S. J., \& Roe, P. L, Improved simulation of flow regime transition in sewers: Two-component pressure approach, Journal of hydraulic engineering, 2006, 132(6), 553562

[5] Vasconcelos, J. G., \& Wright, S. J, Comparison between the two-component pressure approach and current transient flow solvers, Journal of Hydraulic Research, 2007, 45(2), 178-187.

[6] Sanders, B. F., Bradford, S. F, Network implementation of the two-component pressure approach for transient flow in storm sewers, Journal of hydraulic engineering, 2010, 137(2), 158-172.

[7] A. Harten, P.D. Lax, B. vanLeer, On upstream differencing and Godunov-type schemes for hyperbolic conservation-laws, SIAM review, 1983, 25(1), 35-61. 